\title{
Untangling a case of painful neuropathy
}

S Keddie ${ }^{1}$, J Fehmi ${ }^{2}$, Z Jaunmuktane ${ }^{3}, D^{\prime}$ Sa $S^{4}$, Stevens $J^{5}$, MP Lunn ${ }^{1}$

1. MRC Centre for Neuromuscular Diseases, National Hospital of Neurology and Neurosurgery and Department of Molecular Neuroscience, UCL Institute of Neurology, Queen Square, London, UK.

2. Department of Clinical Neurosciences, West Wing, John Radcliffe Hospital, Oxford, UK

3. Division of Neuropathology, National Hospital of Neurology and Neurosurgery, UCL Hospitals NHS Foundation Trust, Queen Square, London, UK.

4. Cancer Division, University College London Hospitals NHS Foundation Trust, London, UK

5. Department of Neurology, North Bristol NHS Trust

Word count: 1600

Tables: 2

Figures: 1

References: 3

Funding: Stephen Keddie is funded by the Guarantors of Brain and the Association of British Neurologists. Michael Lunn is supported by the National Institute for Health Research, University College London Hospitals, Biomedical Research Centre

Conflicts of interest: None 
A 51-year-old right handed male developed a sensory neuropathy with the sensation of walking on pebbles, and tight cramping pains in the calves requiring amitriptyline. Two months following he became unsteady with difficulty walking downstairs necessitating a stick, with associated fatigue and weight loss of half a stone. He has a history of haemophilia type $A$ and liver cirrhosis from hepatitis $C$ contracted through the transfusion of blood products in the 1980s.

Examination revealed a distal symmetrical sensorimotor polyneuropathy with MRC power grade $4+/ 5$ in the intrinsic hand muscles and 0/5 in ankle plantar and dorsiflexion. Supinator, knee and ankle deep tendon reflexes were all absent, with mute plantar responses. He had loss of pinprick sensation to the knees, vibration loss to the costal margins and temperature perception altered to the mid thighs. Dusky pigmentation of the skin and peripheral oedema to the lower limbs were noted.

What is the differential diagnosis, and which investigations should be considered at this stage?

This patient's history and examination findings are typical of a distal symmetrical neuropathy (see table 1 for the differential diagnosis of a painful large fibre neuropathy). Serological workup for a neuropathy should include full blood count, B12, folate, ESR, glucose, thyroid function, a serum protein electrophoresis and immunofixation and possibly ANA. Note that it is typical for hospital laboratories to only perform serum immunofixation if the serum protein electrophoresis is abnormal. The greater sensitivity of immunofixation may reveal low level paraproteins otherwise undetectable, which may be highly relevant in the context of neuropathy. This patient also has a history of hepatitis C, is associated with cryoglobulinaemia and cryoglobulinaemic peripheral nerve vasculitis, and therefore an important differential. Constitutional symptoms including weight loss are uncommon in isolated peripheral neuropathy and therefore systemic causes should be considered, 
including vasculitis, malignancies, metabolic (diabetes), infective and amyloidosis. Thus, a vasculitic screen, LDH, HIV test, antineuronal and antiganglioside antibodies may also be useful.

Nerve conduction and EMG are necessary to characterise the distribution and pattern of motor and sensory involvement, including differentiating demyelinating from axonal pathologies.

Neurophysiology displayed a symmetrical length dependent sensorimotor neuropathy with slowed conduction velocity (CV) in the upper limb, delayed F waves and axonal loss in the lower limbs, and denervation (table 2). Slowed CV and prolonged F-waves are suggestive of a demyelinating disorder, of which CIDP is most common, but this remains a diagnosis of exclusion. Other causes include genetic, drugs (amiodarone, immunomodulatory, immunosuppressive and antineoplastic agents), infective and paraprotein-associated neuropathies. This degree of axonal loss would be very atypical for CIDP at an early stage (unless in nodo-paranodopathies with reversible conduction failure), and given it can result in conduction slowing as a secondary phenomenon from preferential large fibre loss, one should seek clarification from neurophysiology colleagues as to whether the conduction slowing recorded is explained by the degree of axonal loss, or representative of 'true' slowing.

\section{Blood tests revealed type I cryoglobulinaemia. What is the significance of this result and the likelihood of causing neuropathy?}

Cryoglobulinaemia is the presence of serum immunoglobulins that precipitate in vitro at temperatures below $37^{\circ} \mathrm{C}$ and re-dissolve after warming. Cryoprecipitate type is relevant to the diagnosis and is determined through immunofixation of the re-dissolved cryoprecipitate. Type I consists of monoclonal immunoglobulin, either IgM or IgG, can be associated with hyperviscosity syndrome, and can cause vascular occlusion. Type II and III are 'mixed', with a combination of monoclonal IgM and polyclonal IgG, or polyclonal IgM and IgG. Cryoglobulins are pathogenic through the formation of immune complexes which bind to receptors on epithelial cells causing vascular 
inflammation. The leading cause of mixed cryoglobulinaemia is chronic hepatitis $\mathrm{C}$ infection, but other infections i.e. HIV and hepatitis B, and autoimmune diseases, such as Sjögren's syndrome, systemic lupus erythematosus (SLE) and rheumatoid arthritis are also associated. Multi-system clinical manifestations result from cryoglobulin precipitation and vascular inflammation in target organs i.e. skin (ulcers, gangrene, livedo reticularis, purpuric rash and others), vasomotor (Raynaud's phenomenon and acrocyanosis), peripheral or central nervous system, musculoskeletal (arthralgias and arthritis), and renal (renal failure, proteinuria). ${ }^{1}$

Neurological involvement is seen in approximately $30 \%$ of patients with type I cryoglobulins, with $20-60 \%$ of patients presenting with peripheral neuropathy as the initial symptom. ${ }^{1}$ Presentation is predominantly with a sensory neuropathy affecting both large and small fibres, resulting in painful parasthaesia most commonly in the lower limbs, which can be asymmetric and is typically worse at night. Less commonly patients present with a multiple mononeuropathy, typified by a painful asymmetric, sequential peripheral nerve impairment. Motor symptoms do occur but often later.

This patient's cryoglobulin immunofixation demonstrated type I cryoglobulins, which are monoclonal and therefore typically driven by a B-cell haematological malignancy such as B-cell lymphoma, multiple myeloma, Hodgkin's lymphoma or chronic lymphocytic lymphoma. An IgG lambda paraprotein was discovered. Paraproteins are present in approximately $10 \%$ of patients with neuropathy with no other cause, though the presence of a demyelinating neuropathy with any paraprotein should warrant further investigation to determine its origin and potential pathogenic role.

\section{What investigations are necessary following the identification of a monoclonal gammopathy?}

A monoclonal gammopathy should be investigated to delineate its origin as monoclonal gammopathy of undetermined significance (MGUS), lymphoma, the lymphocytic leukaemias, 
multiple myeloma or light chain amyloidosis. MGUS is of considerable clinical importance at diagnosis due to an $18 \%$ risk of progression to myeloma or other plasma or lymphoid malignancy in 20 years, and its known causal association with several serious non-malignant disorders, including anti-MAG neuropathy, paraprotein-associated neuropathy, POEMS syndrome and CANOMAD. Isolated, low-risk MGUS patients do not necessitate bone marrow biopsy or skeletal imaging. The Mayo Clinic risk stratification model define low risk as serum $M$ protein $<15 \mathrm{~g} / \mathrm{l}$, IgG isotype and normal serum free light chain ratio. In such patients, the probability of finding disease necessitating haematological treatment was $4.7 \%{ }^{2}$ This case can be considered a 'monoclonal gammopathy of neurological significance' in that the MGUS may be contributing to cause neuropathy (see box 1 for differentials). An MGUS which does not fit the clinical or immunological phenotypes of the diagnoses listed in box 1 is likely to be coincidental to the neuropathy than causative. Anti-MAG antibodies (in IgM cases), antiganglioside antibodies (including anti-disialosyl in CANOMAD), skeletal imaging and bone marrow biopsy would be necessary investigations. Skeletal imaging can be performed by X-ray, CT or now whole body MRI scan, but FDG-PET CT or MRI scanning may be of more clinical utility, and can demonstrate bone lesions, plasmacytoma and peripheral nerve neurolymphomatosis. Serum vascular Endothelial Growth Factor (sVEGF) is a proinflammatory cytokine often elevated in POEMS syndrome and therefore an important diagnostic biomarker.

A skeletal survey and MRI whole spine were performed in this case, demonstrating sclerotic sacral and pelvic bone lesions thought to represent bony metastases or myeloma. Bone marrow examination revealed only $3 \%$ plasma cells, which were occasionally binucleated and therefore atypical and likely clonal. Bone lesion biopsy was consistent with a plasmacytoma.

Serum vascular endothelial growth factor (sVEGF) was taken, which was $850 \mathrm{pg} / \mathrm{ml}$ (ULN 771 pg/ml). 


\section{What is the significance of the VEGF result?}

SVEGF is often in the several thousands in patients with POEMS. Levels over $771 \mathrm{pg} / \mathrm{ml}$ and under $1500 \mathrm{pg} / \mathrm{ml}$ are more difficult to interpret. It is an extremely sensitive diagnostic test for POEMS syndrome, with a sensitivity of $100 \%$ and specificity of $92 \%$ in patients with demyelinating neuropathy and lambda light chain associated paraproteinaemia, but was only mildly elevated in this case. Given SVEGF can be raised in cases of vasculitic neuropathy and other clonal haematological disorders, this result is not diagnostic. Other false positive causes of raised sVEGF are iron deficiency anaemia and chronic hypoxia secondary to COPD or sleep apnoea.

At this stage, the patient has a clonal plasma cell proliferative disorder, demonstrated both in the definite plasmacytoma and likely in the bone marrow, combined with a painful sensorimotor neuropathy. From the investigations it remained unclear whether the patient's neuropathy was a result of cryoglobulinaemic peripheral nerve vasculitis, or secondary to POEMS syndrome.

\section{Which test would be useful to rule in/out cryoglobulinaemic vasculitis?}

A sural nerve biopsy was performed which showed depletion of large myelinated fibres, widespread active axonal degeneration and occasional regeneration without any histopathological evidence of cryoglobulinaemic vasculitis, although given the patchy nature of vasculitis, it may be missed. Electron microscopy showed evidence of uncompacted myelin lamellae, which is suggestive but not specific for POEMS syndrome (figure 1). Although the cryoglobulinemia was a useful clue towards the presence of a haematological malignancy, the cryoglobulins were not deemed to be the cause of neuropathy.

Thus, a diagnosis of POEMS was made on the basis of the typicalclinical and neurophysiological findings, an IgG lambda paraprotein, with associated skin changes (acrocyanosis - dusky red lower limbs), peripheral oedema, sclerotic bone lesions and mildly raised VEGF. The patient was treated 
with a melphalan-200 autologous stem cell bone marrow transplant. Subsequently the paraprotein disappeared and VEGF normalised, in keeping with complete haematological response. Neuropathic pain resolved, and the patient is now walking unaided.

\section{Discussion}

POEMS syndrome is a rare condition typified by the presence of an inflammatory demyelinating neuropathy and a monoclonal plasma cell disorder, which is lambda light chain restricted. Pain in the lower limbs is a common but under-reported early clinical feature, typically described as a cramping sensation in the calves bilaterally, often mistaken for peripheral vascular disease, with peripheral nerve vasculitis as another possible differential. Although a rare association of an already rare disease, type I cryoglobulinaemic neuropathy has been cited in POEMS syndrome. ${ }^{3}$

Very often, if a positive VEGF is discovered in the correct clinical context (typical neuropathy, lambda light chain paraprotein +/- other POEMS features), POEMS is highly likely, and nerve biopsy would not be necessary to investigate the cause once typical bone marrow +/- plasmacytoma histology demonstrates an abnormal plasma cell clone. However, in this case, a nerve biopsy was crucial to ascertain the cause of neuropathy where the differential diagnosis was of POEMS syndrome versus cryoglobulinaemic neuropathy of an alternative cause.

Nerve biopsy in POEMS syndrome displays loss of large and small myelinated fibre density. Electron microscopy demonstrating uncompacted myelin lamellae is suggestive of POEMS syndrome, present in $60-80 \%$ of POEMS nerve biopsies. There were no features suggestive of vasculitis, and thus the histopathological features of neuropathy was of POEMS syndrome rather than cryoglobulinaemia. 
Key points

- Severe axonal loss on neurophysiology is atypical in early CIDP (unless associated with nodoparanodopathies) and alternative diagnoses should be considered.

- Serum immunofixation is more sensitive to protein electrophoresis and may uncover a small but potentially neurologically significant paraprotein.

- Anti-MAG antibodies (in IgM cases), antiganglioside antibodies (including anti-disialosyl in CANOMAD), VEGF, skeletal imaging and bone marrow biopsy would be necessary investigations for a potential monoclonal gammopathy of neurological significance.

- Serum VEGF is a highly sensitive and specific biomarker for POEMS syndrome, but can be mildly raised in vasculitic neuropathies., iron deficient anaemia and chronic hypoxia. 
Table 1- Differential diagnosis of a large fibre painful peripheral neuropathy

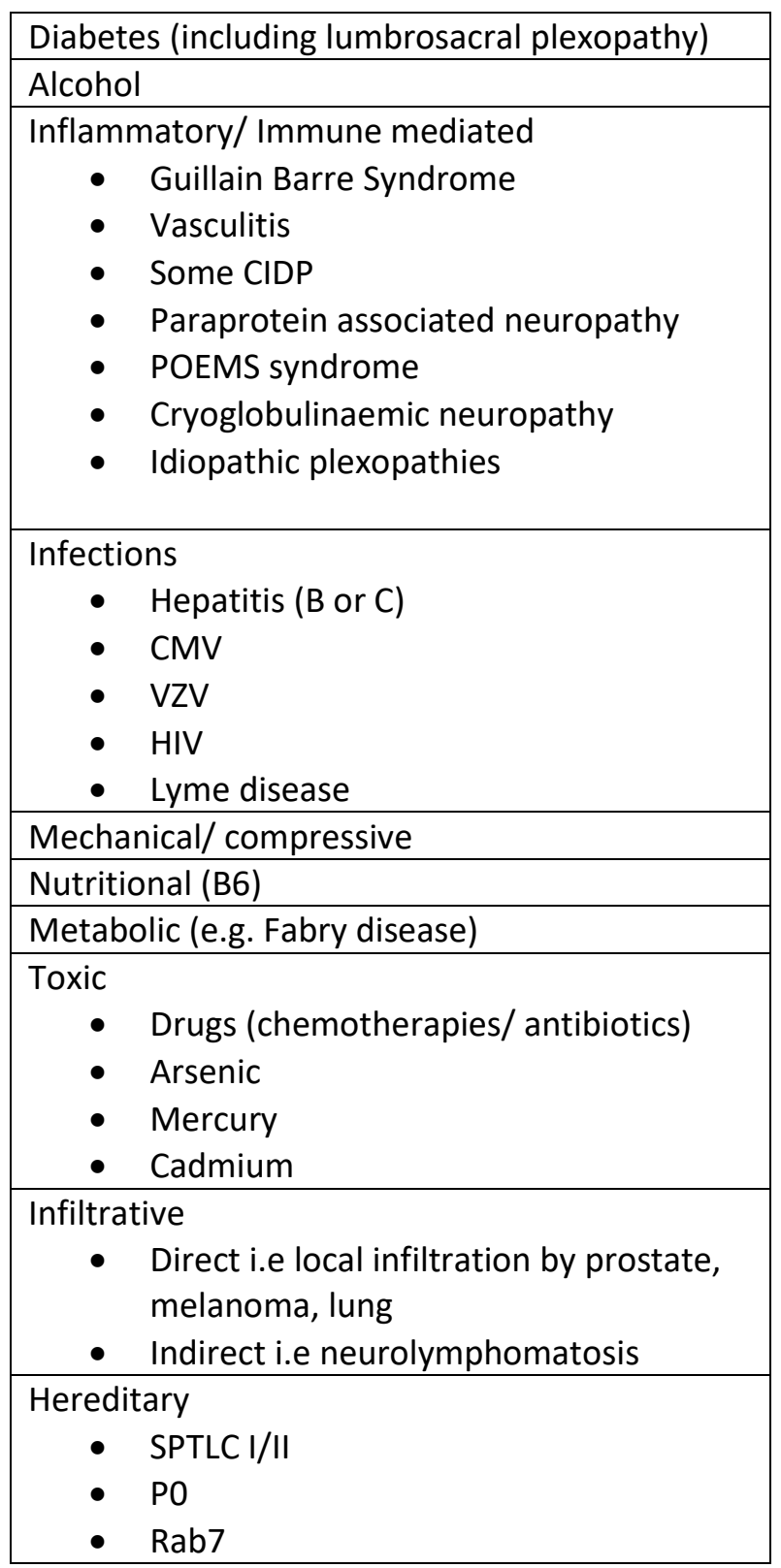


Table 2 - Neurophysiology and EMG results

\begin{tabular}{|l|l|l|}
\hline \multirow{2}{*}{ Sensory nerve conduction studies } & \multicolumn{2}{|c|}{} \\
\cline { 2 - 3 } & $\mu V$ & $\mathrm{~m} / \mathrm{s}$ \\
\hline Right Radial - Thumb- Forearm & 12.6 & 44.9 \\
\hline Left Radial - Thumb- Forearm & 6.3 & 40.7 \\
\hline Right median- Digit II- Wrist & 7.3 & 42.9 \\
\hline Right sural- Lat malleolus- Calf & Not recordable & Not recordable \\
\hline $\begin{array}{l}\text { Left superficial personal- Foot- Lateral } \\
\text { leg }\end{array}$ & Not recordable & Not recordable \\
\hline
\end{tabular}

\begin{tabular}{|l|l|l|l|l|}
\hline \multirow{2}{*}{$\begin{array}{l}\text { Motor nerve } \\
\text { conduction studies }\end{array}$} & \multicolumn{2}{|l|}{$\mathrm{DML}(\mathrm{ms})$} & $\mathrm{CMAP}(\mathrm{mV})$ & $\begin{array}{l}\text { Minimal F- } \\
\text { wave latency }\end{array}$ \\
\cline { 2 - 5 } & $\mathrm{ms}$ & $\mathrm{m} / \mathrm{s}$ & $\mathrm{mV}$ & $\mathrm{ms}$ \\
\hline Right Median- APB & \multicolumn{5}{|l|}{} \\
\hline 1. Wrist & 3.75 & & 10.3 & \\
\hline 2. Elbow & 11.00 & 36.6 & 8.0 & \\
\hline 3. Axilla & 15.45 & 31.5 & 9.3 & \\
\hline $\begin{array}{l}\text { Right Common } \\
\text { Peroneal - EDB }\end{array}$ & Not recordable & & & \\
\hline Right Tibial - AH & Not recordable & & & \\
\hline Left Tibial- AH & Not recordable & & & \\
\hline
\end{tabular}

\begin{tabular}{|l|l|}
\hline \multirow{2}{*}{ F- Wave } & $\begin{array}{l}\text { Mean F-wave } \\
\text { latency (ms) }\end{array}$ \\
\cline { 2 - 2 } & \\
\hline Right median- APB & 52.49 \\
\hline Right ulnar- ADM & 49.56 \\
\hline Left ulnar- ADM & 54.86 \\
\hline Left median- APB & 52.96 \\
\hline
\end{tabular}

\begin{tabular}{|l|l|l|l|l|l|}
\hline EMG & Fibs & Duration & Amplitude & Polyphasic & Recruitment \\
\hline R biceps & 0 & 0 & $\mathrm{~N}$ & $\mathrm{~N}$ & $\mathrm{~N}$ \\
\hline R FDIO & 0 & 0 & $\mathrm{~N}$ & $\mathrm{~N}$ & $\mathrm{~N}$ \\
\hline R Gastrocnemius & 1 & 0 & Increased & Increased & $\begin{array}{l}\text { Reduced, } \\
\text { large units } \\
\text { recruiting } \\
\text { early }\end{array}$ \\
\hline R vastus & 2 & 0 & Increased & Increased & $\begin{array}{l}\text { Not } \\
\text { assessable }\end{array}$ \\
\hline
\end{tabular}


Box 1- The differential of 'monoclonal gammopathy of neurological significance'

\section{Differential of 'monoclonal gammopathy of neurological significance'}

Paraprotein associated neuropathy causing distal acquired demyelinating neuropathy with or without anti MAG antibodies (IgM)

Paraprotein associated neuropathy with CIDP phenotype (more likely IgA or IgG)

POEMS syndrome (Polyneuropathy, Organomegaly, Endocrinopathy, Monoclonal gammopathy, Skin) (IgA or IgG lambda light chain restricted)

CANOMAD (Chronic sensory ataxic neuropathy with anti-disialosyl antibodies) (IgM)

Neurolymphomatosis (IgG, IgA or IgM)

Light chain amyloidosis (typically lambda)

Coincidental finding to the neuropathy 


\section{References}

1. Dalton JA, Higgins MK, Miller AH, Keefe FJ, Khuri FR. Clinical presentation and outcomes of patients with type 1 monoclonal cryoglobulinemia. 2016;38(5):457-464.

2. Rajkumar S V., Kyle RA, Therneau TM, et al. Serum free light chain ratio is an independent risk factor for progression in monoclonal gammopathy of undetermined significance. Blood. 2005;106(3):812-817.

3. Iqbal M, Bilal S, Hennessy M. POEMS syndrome: cryoglobulinemia an unusual association. Ir J Med Sci. 2011;180(3):741-743. 